\title{
Dificuldades na identificação laboratorial da talassemia alfa
}

\author{
Difficulty on laboratory identification of alpha thalassemia
}

Karlla Greick Batista Dias-Penna'; Paulo Roberto de Melo-Reis²; Mauro Meira de Mesquita ${ }^{3}$; Jairo Batista da Silva ${ }^{4}$; Luiz Artur Mendes Bataus ${ }^{5}$

\begin{abstract}
unitermos
Hemoglobinopatias

Talassemia alfa

Hemoglobina H

\section{resumo}

Introdução: Talassemia alfa é uma síndrome associada à redução da síntese de cadeias de globina do tipo alfa. A gravidade das manifestações clínicas está relacionada com a quantidade de globinas produzida e a estabilidade das cadeias beta presentes em excesso. A talassemia alfa mínima resulta da deleção de apenas um dos quatro genes $\alpha(-\alpha / \alpha \alpha)$. Clinicamente apresenta anemia leve com microcitose ou ausência de anemia, sendo o diagnóstico realizado por meio de visualização da hemoglobina $(\mathrm{Hb}) \mathrm{H}$ por eletroforese alcalina em acetato de celulose ou por identificação de inclusões celulares de $\mathrm{Hb} \mathrm{H}$ coradas pelo azul de crezil brilhante. Objetivo: Avaliar portadores de talassemia alfa e seus respectivos progenitores, correlacionando perfil hematológico e presença de $\mathrm{Hb} \mathrm{H}$, utilizando procedimentos laboratoriais clássicos em três diferentes amostragens. Discussão e conclusão: Os dados obtidos mostram que a presença de $\mathrm{Hb} \mathrm{H}$, indicativo de talassemia alfa, pode não ser confirmada em uma análise posterior. Entre os fatores que podem influenciar no não aparecimento de $\mathrm{Hb} \mathrm{H}$ em pessoa comprovadamente com talassemia alfa está a deficiência de ferro. A talassemia alfa está associada a defeitos envolvendo os genes codificadores da cadeia alfa, mas também pode estar relacionada com desbalanciamento temporário na expressão dos genes globina, diminuição de alfa ou aumento de beta, o que poderia explicar o aparecimento de tetrâmeros de cadeia beta $(\mathrm{Hb} \mathrm{H})$, sugerindo diagnóstico de talassemia alfa mínima.
\end{abstract}

Introduction: Alpha thalassemia is a syndrome with associated with the reduction of alpha globin chain synthesis. The severity of clinical manifestations is related to the amount of globins produced and the stability of beta chains that are present in excess. Alpha thalassemia minor is caused by the deletion of one of the four genes $\alpha(-\alpha / \alpha \alpha)$. Clinically, it presents mild anemia with microcytosis or absence of anemia. The diagnosis is made by the visualization of $\mathrm{Hb} \mathrm{H}$ through alkaline electrophoresis on cellulose acetate or by the identification of inclusion bodies stained with brilliant cresyl blue. Objective: Evaluate alpha thalassemia carriers and their respective progenitors, correlating their hematology profile and the presence of $\mathrm{Hb} \mathrm{H}$ by means of standard laboratory procedures in three different samplings. Discussion and conclusion: The results show that the presence of $\mathrm{Hb} \mathrm{H}$, which is indicative of alpha thalassemia, may not be confirmed in a subsequent analysis. Iron deficiency in $\mathrm{Hb} \mathrm{H}$ carriers is among the factors that may influence on the absence of $\mathrm{Hb} \mathrm{H}$ in alpha thalassemia proven patients. Alpha thalassemia is associated with genetic defects involving alpha chain encoding genes, but may be also associated with a temporary imbalance of globin gene expression, alpha chain reduction or beta increase, which could explain the presence of beta chain tetramer $(\mathrm{Hb} H)$ leading to the diagnosis of alpha thalassemia minor.

1. Mestra em Biologia (áreas de concentração: Bioquímica e Biologia Celular) pela Universidade Federal de Goiás (UFC); professora de Biologia Molecular e Fisiologia Humana do Departamento de Biomedicina da Pontifícia Universidade Católica de Góias (PUC-Goiás); doutoranda em Biologia Celular e Molecular pela UFG.

2. Mestre em Ciências Ambientais e Saúde pela PUC-Goiás; professor assistente do Departamento de Biomedicina da PUC-Coiás.

3. Mestre em Ciências Ambientais e Saúde pela PUC-Coiás; professor assistente do Departamento de Biomedicina da PUC-Coiás.

4. Mestre em Ciências Ambientais e Saúde pela PUC-Coiás; professor assistente do Departamento de Biomedicina da PUC-Goiás.

5. Doutor em Biologia pela Universidade de Brasília (UnB); professor do Departamento de Biologia do Instituto de Ciências Biológicas (ICB) da UFC. 


\section{Introdução}

A talassemia (hemoglobinopatia) é uma síndrome que envolve genes produtores de globina, alfa e não alfa (beta, delta e gama), encontrada em vários países, representando sério problema de saúde global, principalmente na Europa, na África e na Ásia(23). No Brasil, devido a grande migração de povos originados desses continentes, é descrita com significativa frequência de hemoglobinopatias, reflexo dessa enorme diversidade étnica, dependendo da região estudada. Relatos das décadas de 1970 e 1980 mostraram existência de pacientes portadores de talassemia alfa no Brasil(15, 24).

A talassemia alfa está associada a síntese reduzida de cadeias de globina alfa e consequente aumento de concentração de cadeias beta. Esse desbalanceamento induz a formação de tetrâmeros de cadeias beta denominados hemoglobina $(\mathrm{Hb}) \mathrm{H}^{(22)}$. Esses tetrâmeros apresentam instabilidade que resulta na precipitação dessa hemoglobina sobre a membrana do eritrócito, diminuindo consequentemente a sobrevida das hemácias ${ }^{(32)}$. Atualmente, cerca de 4.516 publicações sobre talassemia alfa estão disponíveis no banco de dados de PubMed, serviço da U. S. National Library of Medicine (NLM) ${ }^{(13)}$.

Nos cromossomos 11 e 16 estão presentes os complexos gênicos responsáveis pela síntese de cadeias globínicas alfa $(\alpha)$, beta $(\beta)$, gama $(\gamma)$, delta $(\delta)$, épsilon $(\varepsilon)$ e zeta $(\zeta)$. Em região de aproximadamente $30 \mathrm{~kb}$ do cromossomo 16 (16p13.3) está o cluster $\alpha 5^{\prime}-\zeta-\psi \zeta-\psi \alpha 2-\psi \alpha 1-\alpha 2-\alpha 1-\theta 1-3^{\prime}$ e no cromossomo 11 (11p15.5) está o cluster $\beta 5^{\prime}-\varepsilon-\mathrm{C} \gamma$ $A \gamma-\psi \beta-\delta-\beta-3^{\prime}$, que compreende aproximadamente região de $60 \mathrm{~kb}^{(22,25)}$. A partir do sexto mês após o nascimento, a molécula de $\mathrm{Hb}$ predominante é designada como $\mathrm{Hb}$ A ( $\alpha 2 \beta 2)$, representando de $95 \%$ a $98 \%$ do total de hemoglobinas. Em menor quantidade, de $2,0 \%$ a $4,0 \%$, existe a $\mathrm{Hb} \mathrm{A}_{2}(\alpha 2 \delta 2)$ e, com menos de $1,0 \%$, a $\mathrm{Hb}$ fetal $(\alpha 2 \gamma 2)^{(23,25)}$.

As manifestações clínicas da talassemia alfa são bastante diversificadas dependendo do número de genes alfa afetados. Existem quatro genes alfa ativos representados por dois haplótipos $\alpha \alpha(\alpha \alpha / \alpha \alpha)$. Assim, talassemias alfa são classificadas em: portador silencioso ou $\alpha$-talassemia mínima, que resulta da deleção de um dos quatro genes $\alpha$ $(-\alpha / \alpha \alpha)$; traço $\alpha$-talassêmico ou $\alpha$-talassemia menor, que resulta da deleção de dois genes $\alpha$ no mesmo cromossomo $(--/ \alpha \alpha)$ ou em cromossomos diferentes $(-\alpha /-\alpha)$; doença da $\mathrm{Hb} \mathrm{H}$ ou $\alpha$-talassemia intermédia, em que três genes foram deletados $(--/-\alpha)$; e hidropsia fetal, que resulta da deleção dos quatro genes $\alpha(--/--)^{(25)}$.
A regulação dos genes alfa se faz por meio de região conhecida como HS-40, localizada a $40 \mathrm{~kb}$ upstream do gene $\zeta$, e sua integridade é essencial para expressão dos genes do cluster. As deleções que removem essa sequência silenciam a expressão dos genes do cluster alfa localizado em posição downstream ${ }^{(6)}$.

O diagnóstico de talassemia alfa pode ser realizado pela detecção de $\mathrm{Hb} \mathrm{H}$ no sangue hemolisado, por meio de eletroforese alcalina em acetato de celulose ou de visualização dos corpos de inclusão de $\mathrm{Hb} \mathrm{H}$ corados com azul de crezil brilhante nos eritrócitos do sangue periférico ${ }^{(23)}$. Segundo Skogerboe et al. ${ }^{(11)}$ (1992) e Thompson et al. ${ }^{(7)}$ (1989) existe correlação entre formação dos corpos de inclusão de $\mathrm{Hb}$ $\mathrm{H}$ e os vários genótipos alfa, no entanto sua ausência não exclui o diagnóstico, principalmente em caso de portador silencioso ou $\alpha$-talassemia mínima.

Este trabalho teve como objetivo avaliar portadores de talassemia alfa, correlacionando perfil hematológico e presença de $\mathrm{Hb} \mathrm{H}$, utilizando procedimentos laboratoriais clássicos em três diferentes amostragens. Foram analisadas também amostras dos progenitores desses portadores.

\section{Casuística e métodos}

Foi realizado acompanhamento dos resultados de indivíduos que apresentaram migração eletroforética compatível com $\mathrm{Hb} \mathrm{H}$ na eletroforese alcalina de hemoglobina em acetato de celulose em três amostragens diferentes. $O$ estudo foi realizado no Laboratório de Estudo e Pesquisa de Anemias Hereditárias (LEPAH) do Departamento de Biomedicina da Universidade Católica de Goiás (UCG), de acordo com normas da resolução número 196/98 do Conselho Nacional de Saúde (CNS). As amostras foram coletadas em 2001, 2004 e 2006, compreendendo período de seis anos. Foram analisadas 30 amostras de sangue coletadas com ácido etilenodiaminotetracético (EDTA), provenientes de 10 crianças de ambos os sexos e de seus respectivos pais. $\mathrm{O}$ trabalho faz parte de projeto submetido e aprovado pelo comitê de ética da Universidade Federal de Goiás (UFG) sob protocolo número 065/2005.

Para determinação da talassemia alfa foi realizada pesquisa de $\mathrm{Hb} \mathrm{H}$ por meio de eletroforese em acetato de celulose em pH alcalino (tampão tris-EDTA-ácido bórico [TEB], $\mathrm{pH}=8,5)$ com hemolisado rápido, utilizando saponina a $1 \%{ }^{(10)}$ e detecção dos corpos de inclusão de $\mathrm{Hb} \mathrm{H}$ (PIEHH) com azul de crezil brilhante ${ }^{(10,14)}$.

Foram realizadas, também, resistência globular osmótica em cloreto de sódio a 0,36\% ${ }^{(16)}$ e avaliação de volume 
corpuscular médio (VCM) dos eritrócitos pelo equipamento Pentra $60^{\circledast}(\mathrm{ABX})$, adotando valor de referência de 80 a $86 \mathrm{fL}$ para ambos os $\operatorname{sexos}^{(25)}$.

As amostras que apresentaram $\mathrm{Hb} \mathrm{S}$ foram submetidas para confirmação ${ }^{(25)}$ aos testes de eletroforese ácida de hemoglobinas, falcização com solução de metabissulfito de sódio a $2 \%$ e cromatografia líquida de alta resolução (HPLC) no aparelho Variant ${ }^{\circledR}$ da Bio-Rad.

\section{Resultados}

O perfil dos tipos de hemoglobinas presentes no sangue de 10 crianças e de seus respectivos pais foram avaliados por eletroforese alcalina em acetato de celulose. As amostras foram coletadas em diferentes épocas (2001, 2004 e 2006) e são mostradas na Tabela 1.

Oito entre as 10 crianças identificadas como portadoras de $\mathrm{Hb} \mathrm{H}$ na primeira avaliação apresentaram variação quanto à presença dessa hemoglobina quando reavaliadas. Três crianças não apresentaram mais perfil de $\mathrm{Hb} \mathrm{H}$. Apenas as crianças 5 e 8 mantiveram o mesmo perfil da primeira amostragem. Dos 20 pais avaliados, 11 não apresentaram $\mathrm{Hb} \mathrm{H}$ em nenhuma das avaliações. Nove pais apresentaram $\mathrm{Hb} \mathrm{H}$, sendo que cinco apresentaram alternância de resultado. Ao todo foram avaliados 30 indivíduos, 13 desses apresentando resultados contraditórios quanto à presença de $\mathrm{Hb} \mathrm{H}$, perfazendo total de $43,33 \%$.

Na Tabela 1 pode-se observar também que na amostragem inicial de 2001 todas as crianças avaliadas apresentaram corpos de inclusão de Hb H. Em 2004 e 2006, oito das dez crianças analisadas tiveram resultados contraditórios relativo à PIEHH. Dessas oito crianças, três não apresentaram mais resposta positiva, cinco obtiveram alternância de resultados e apenas duas mantiveram resultado positivo. Dos 20 pais, 12 apresentaram resposta negativa para a PIEHH, fato considerado normal. Quatro pais tiveram resultado positivo em todas as avaliações, três em duas ocasiões e um apenas uma vez. Ao todo, 12 indivíduos apresentaram resultados contraditórios, totalizando $40 \%$.

Observamos, ainda, na Tabela 1 resultados de prova de resistência osmótica à salina $0,36 \%$, na qual sete das 10 crianças analisadas tiveram resultados discordantes. Das sete crianças, três não apresentaram mais resposta positiva. Quatro crianças obtiveram alternância de resultados e apenas três mantiveram o resultado, sendo que as crianças 5 e 8 continuaram com resultado positivo e a criança 10 com negativo. Com relação aos pais, 12 apresentaram res- posta negativa para a prova de resistência osmótica à salina $0,36 \%$, resultado considerado normal. Três pais apresentaram resistência em todas as avaliações, três em duas e dois somente uma vez. Ao todo doze indivíduos apresentaram resultados contraditórios, completando total de $40 \%$.

Os indivíduos que apresentaram $\mathrm{Hb} S$ mantiveram mesmo resultado em todos os testes confirmatórios: análise eletroforética em $\mathrm{pH}$ ácido, análise cromatográfica utilizando HPLC e falcização positiva com metabissulfito de sódio.

Morfologia e volume corpuscular médio dos eritrócitos (VCM) apresentaram grande variação entre as três amostragens (Tabela 2). Adotando valor de $80 \mathrm{fL}$ como limite entre normocitose e microcitose, pode-se observar que oito dos 20 pais apresentaram microcitose em alguma das amostras. Desses oito pais, quatro tiveram microcitose em pelo menos duas avaliações, sendo todos do sexo feminino. Das crianças avaliadas, todas apresentaram VCM com valores inferiores a $80 \mathrm{fL}$ na primeira verificação. Seis crianças tiveram novamente resultados inferiores a $80 \mathrm{fL}$ e duas crianças apresentaram o VCM diminuído nas três amostragens.

\section{Discussão}

Talassemia alfa é caracterizada por redução na produção de cadeias polipeptídicas do tipo alfa que resulta em eritócitos microcíticos e hipocrômicos. A gravidade dessa doença está associada ao grau de comprometimento da expressão da cadeia alfa e pode ser classificada clinicamente em portador silencioso ou talassemia alfa mínima, traço alfa-talassêmico ou talassemia alfa menor, doença da $\mathrm{Hb} \mathrm{H}$ ou talassemia alfa intermédia e hidropsia fetal ${ }^{(23)}$.

Os resultados obtidos neste trabalho mostraram grande variação com relação à presença de $\mathrm{Hb} \mathrm{H}$ e ao volume corpuscular médio (VCM) dos eritrócitos, comparando três amostragens realizadas em 2001, 2004 e 2006. Essa diferença alcançou 43,33\% com relação à existência de $\mathrm{Hb} \mathrm{H}$, como pode ser visto nas Tabelas 1 e 2.

Na primeira avaliação do perfil eletroforético, em 2001, todas as crianças apresentaram $\mathrm{Hb} \mathrm{H}$ (assim como pelo menos um dos pais), com exceção da criança 8, cujos pais não apresentaram essa hemoglobina em avaliação alguma (Tabela 1). É importante ressaltar que a forma mais comum de talassemia alfa é hereditária, entretanto existem formas adquiridas secundárias à patologia primária(22).

Todas as amostras que tiveram $\mathrm{Hb} \mathrm{H}$ na eletroforese alcalina apresentaram corpos de inclusão de $\mathrm{Hb} \mathrm{H}$ na coloração com azul de crezil brilhante e microcitose característica da 
Resultados de perfil eletroforético em acetato de celulose de pesquisa dos corpos de inclusão de $\mathrm{Hb}$ H (PIEHH) e de prova de resistência osmótica à salina 0,36\% dos 30 indivíduos nas amostras Tabela 1 coletadas em 2001, 2004 e 2006*

\begin{tabular}{|c|c|c|c|c|c|c|c|c|c|c|}
\hline \multirow[b]{2}{*}{ Indivíduo } & \multirow[b]{2}{*}{$\begin{array}{c}\text { Idade em } \\
2001\end{array}$} & \multicolumn{3}{|c|}{$\begin{array}{c}\text { Perfil } \\
\text { eletroforético }\end{array}$} & \multicolumn{3}{|c|}{$\begin{array}{c}\text { Pesquisa intraeritrocitária } \\
\text { Hb H }\end{array}$} & \multicolumn{3}{|c|}{$\begin{array}{c}\text { Prova de resistência } \\
\text { osmótica à salina 0,36\% }\end{array}$} \\
\hline & & 2001 & 2004 & 2006 & 2001 & 2004 & 2006 & 2001 & 2004 & 2006 \\
\hline 1 & 02 anos & ASH & AS & ASH & Positivo & Negativo & Positivo & Positivo & Negativo & Positivo \\
\hline $1 P$ & 36 anos & $\mathrm{AH}$ & $\mathrm{AH}$ & $A A$ & Positivo & Positivo & Negativo & Positivo & Positivo & Negativo \\
\hline $1 \mathrm{M}$ & 32 anos & AS & AS & AS & Negativo & Negativo & Negativo & Negativo & Negativo & Negativo \\
\hline 2 & 03 anos & ASH & AS & AS & Positivo & Negativo & Negativo & Positivo & Negativo & Negativo \\
\hline $2 \mathrm{P}$ & 42 anos & $\mathrm{AH}$ & $\mathrm{AH}$ & $\mathrm{AH}$ & Positivo & Positivo & Positivo & Positivo & Positivo & Positivo \\
\hline $2 \mathrm{M}$ & 37 anos & AS & AS & AS & Negativo & Negativo & Negativo & Negativo & Negativo & Negativo \\
\hline 3 & 03 anos & $\mathrm{AH}$ & $A A$ & $A A$ & Positivo & Negativo & Negativo & Positivo & Negativo & Negativo \\
\hline $3 P$ & 28 anos & $A A$ & $A A$ & $A A$ & Negativo & Negativo & Negativo & Negativo & Negativo & Negativo \\
\hline $3 \mathrm{M}$ & 25 anos & $\mathrm{AH}$ & $\mathrm{AA}$ & $\mathrm{AH}$ & Positivo & Negativo & Positivo & Positivo & Negativo & Positivo \\
\hline 4 & 04 anos & $\mathrm{AH}$ & $\mathrm{AA}$ & $\mathrm{AH}$ & Positivo & Negativo & Positivo & Positivo & Negativo & Positivo \\
\hline $4 \mathrm{P}$ & 35 anos & AA & AA & AA & Negativo & Negativo & Negativo & Negativo & Negativo & Negativo \\
\hline $4 \mathrm{M}$ & 34 anos & $\mathrm{AH}$ & $\mathrm{AH}$ & $A A$ & Positivo & Positivo & Negativo & Positivo & Positivo & Negativo \\
\hline 5 & 03 anos & ASH & ASH & ASH & Positivo & Positivo & Positivo & Positivo & Positivo & Positivo \\
\hline $5 P$ & 43 anos & $\mathrm{AA}$ & $A A$ & $\mathrm{AA}$ & Negativo & Negativo & Negativo & Negativo & Negativo & Negativo \\
\hline $5 \mathrm{M}$ & 38 anos & ASH & ASH & ASH & Positivo & Positivo & Positivo & Positivo & Positivo & Positivo \\
\hline 6 & 05 anos & ASH & ASH & AS & Positivo & Positivo & Negativo & Positivo & Positivo & Negativo \\
\hline $6 P$ & 44 anos & AA & $\mathrm{AA}$ & $A A$ & Negativo & Negativo & Negativo & Negativo & Negativo & Negativo \\
\hline $6 \mathrm{M}$ & 41 anos & ASH & ASH & ASH & Positivo & Positivo & Positivo & Positivo & Positivo & Positivo \\
\hline 7 & 09 anos & $\mathrm{AH}$ & AA & $A A$ & Positivo & Negativo & Negativo & Positivo & Negativo & Negativo \\
\hline $7 P$ & 39 anos & $\mathrm{AH}$ & $A A$ & $A A$ & Positivo & Negativo & Negativo & Positivo & Negativo & Negativo \\
\hline $7 \mathrm{M}$ & 37 anos & $A A$ & AA & AA & Negativo & Negativo & Negativo & Negativo & Negativo & Negativo \\
\hline 8 & 06 anos & $\mathrm{AH}$ & $\mathrm{AH}$ & $\mathrm{AH}$ & Positivo & Positivo & Positivo & Positivo & Positivo & Positivo \\
\hline $8 P$ & 44 anos & AA & AA & $A A$ & Negativo & Negativo & Negativo & Negativo & Negativo & Negativo \\
\hline $8 \mathrm{M}$ & 43 anos & $\mathrm{AA}$ & AA & $A A$ & Negativo & Negativo & Negativo & Negativo & Negativo & Negativo \\
\hline 9 & 07 anos & $\mathrm{AH}$ & $A A$ & $\mathrm{AH}$ & Positivo & Negativo & Positivo & Positivo & Negativo & Positivo \\
\hline $9 P$ & 45 anos & $A A$ & $A A$ & $A A$ & Negativo & Negativo & Negativo & Negativo & Negativo & Negativo \\
\hline $9 \mathrm{M}$ & 38 anos & AA & AH & AH & Negativo & Negativo & Negativo & Negativo & Negativo & Negativo \\
\hline 10 & 05 anos & $\mathrm{AH}$ & $A A$ & $\mathrm{AH}$ & Positivo & Negativo & Positivo & Negativo & Negativo & Negativo \\
\hline $10 P$ & 37 anos & $\mathrm{AA}$ & AA & $A A$ & Negativo & Negativo & Negativo & Negativo & Negativo & Negativo \\
\hline $10 M$ & 34 anos & $\mathrm{AH}$ & $\mathrm{AH}$ & $\mathrm{AH}$ & Positivo & Positivo & Positivo & Positivo & Positivo & Negativo \\
\hline
\end{tabular}


Resultados do volume corpuscular médio (VCM), em fentolitros (fL), dos

Tabela 2

30 indivíduos

\begin{tabular}{|c|c|c|c|}
\hline Indivíduo & 2001 & 2004 & 2006 \\
\hline 1 & 72,3 & 87,6 & 79,7 \\
\hline $1 P$ & 80,5 & 78,5 & 84,3 \\
\hline $1 \mathrm{M}$ & 88,5 & 90,5 & 90,1 \\
\hline 2 & 75,7 & 88,3 & 86,7 \\
\hline $2 P$ & 81,5 & 80,2 & 78,9 \\
\hline $2 \mathrm{M}$ & 88,5 & 90,8 & 91,4 \\
\hline 3 & 79,4 & 85,7 & 85,7 \\
\hline $3 P$ & 92,1 & 91,1 & 89,6 \\
\hline $3 M$ & 79,4 & 89,7 & 78,3 \\
\hline 4 & 75,5 & 88,3 & 77,2 \\
\hline $4 \mathrm{P}$ & 92,1 & 88,5 & 90,7 \\
\hline $4 M$ & 79,4 & 77,6 & 89,9 \\
\hline 5 & 75,7 & 73,9 & 74,3 \\
\hline $5 P$ & 90,5 & 89,8 & 90,5 \\
\hline $5 M$ & 80,6 & 78,7 & 80,1 \\
\hline 6 & 79,4 & 80,1 & 85,7 \\
\hline $6 P$ & 91,2 & 92,1 & 89,9 \\
\hline $6 \mathrm{M}$ & 81,2 & 78,3 & 77,4 \\
\hline 7 & 76,5 & 84,3 & 87,6 \\
\hline $7 P$ & 78,7 & 89,4 & 89,3 \\
\hline $7 \mathrm{M}$ & 90,6 & 91,4 & 86,4 \\
\hline 8 & 72,7 & 77,5 & 75,6 \\
\hline $8 P$ & 88,6 & 90,3 & 88,6 \\
\hline $8 M$ & 92,2 & 89,8 & 92,2 \\
\hline 9 & 78 & 88,1 & 77,3 \\
\hline $9 P$ & 89,7 & 91,5 & 89,7 \\
\hline $9 M$ & 90,5 & 80,4 & 90,5 \\
\hline 10 & 77,4 & 88,2 & 78,4 \\
\hline $10 P$ & 91,2 & 90,6 & 91,2 \\
\hline $10 M$ & 80,3 & 78,2 & 76,9 \\
\hline
\end{tabular}

P: pai; M: mãe.

talassemia alfa. No entanto, a ausência de corpos de inclusão de Hb H na PIEHH não exclui o diagnóstico(7, 11, 12), uma vez que essa técnica exige muita habilidade e, mesmo em indivíduos com deleção de dois genes alfa, é comum encontrar uma ou duas dessas inclusões após vários minutos duradouros de busca ${ }^{(20)}$. Das crianças estudadas, oito apresentaram variação com relação à presença de $\mathrm{Hb} \mathrm{H}$ nas amostras coletadas em diferentes épocas.

$\mathrm{Na}$ análise de resistência globular à salina $0,36 \%$ somente a criança 10 apresentou teste negativo, porém a $\mathrm{Hb} \mathrm{H}$ foi detectada pela visualização dos corpos de inclusão e pela eletroforese, nas avaliações de 2001 e 2006. Isso acontece porque o teste de triagem desenvolvido por Silvestroni e Bianco $^{(16)}$ (1975) é pouco específico e visa apenas detecção de células microcíticas, dependendo diretamente da quantidade dessas células e de seu volume médio. Com relação aos pais, somente a mãe $9 \mathrm{M}$ apresentou teste negativo em 2004 e 2006 e presença de $\mathrm{Hb} \mathrm{H}$ na eletroforese.

Todas as amostras que apresentaram $\mathrm{Hb} \mathrm{H}$ apresentaram eritrócitos microcíticos e VCM baixo (menor que $80 \mathrm{fL}$ ), demonstrando estreita correlação entre microcitose e presença de $\mathrm{Hb} \mathrm{H}$. Esses dados corroboram o trabalho de Chan et al.(2), que estudaram a importância de se avaliar pessoas com VCM normal em áreas de alta incidência de talassemia, principalmente quando os valores de VCM forem próximos ao limite inferior ( $80 \mathrm{fL}$ ). Nesse estudo, conduzido em Hong Kong, foram estudados 95 indivíduos que apresentavam VCM entre 80 e $85 \mathrm{fL}$ e, desses, 32,63\% apresentaram alfa talassemia.

Os dados obtidos nesse trabalho (Tabela 1) sugerem que o desbalanciamento entre concentrações de cadeias alfa e beta pode ser temporário, visto que ao serem comparadas análises de épocas diferentes encontramos resultados contraditórios para detecção de Hb H. É importante destacar que as talassemias do tipo alfa podem ter duas origens: hereditária e adquirida. As formas hereditárias atingem $4 \%$ da população brasileira, enquanto as formas adquiridas são normalmente secundárias a processo patológico primário ${ }^{(19,23)}$. Alguns autores descrevem interação de hemoglobinas variantes com talassemia alfa. Na anemia falciforme é descrita interação benéfica com talassemia alfa, ocorrendo diminuição da anemia hemolítica, inibição da polimerização intracelular da hemoglobina Se diminuição da intensidade de hemólise ${ }^{(18,19)}$.

Trabalho realizado em nosso laboratório em população estudantil de Goiás encontrou prevalência de 8,2\% de talassemia alfa e $2,1 \%$ de talassemia alfa associada a $\mathrm{Hb} \mathrm{S}$, entretanto não foram investigadas as origens ${ }^{(8)}$. Analisando amostras de indivíduos oriundos de 55 cidades do estado de Goiás detectamos a prevalência de $10,1 \%$ de hemoglobinopatias, 5,2\% de talassemia alfa heterozigótica, $2 \%$ de heterozigose para hemoglobina $\mathrm{S}(\mathrm{Hb} \mathrm{AS}), 1 \%$ de heterozigose para hemoglobina $\mathrm{C}(\mathrm{Hb} \mathrm{AC}), 0,7 \%$ de talassemia beta menor, $0,5 \%$ de associação entre talassemia alfa e heterozigose para $\mathrm{Hb} \mathrm{S,} \mathrm{0,3 \%} \mathrm{de} \mathrm{associação} \mathrm{entre} \mathrm{talassemia}$ 
alfa e heterozigose para $\mathrm{Hb} \mathrm{C}$ e $0,3 \%$ de heterozigose para hemoglobina $\mathrm{D}(\mathrm{Hb} A D)^{(9)}$.

Doenças hematológicas do tipo eritroleucemias, doenças linfo e mieloproliferativas crônicas e agudas e anemia sideroblástica estão normalmente associadas ao aparecimento de talassemia alfa ${ }^{(5,17)}$. Outros tipos de doenças crônicas, tais como doença de Chagas e diabetes mellitus também podem levar a quadro de talassemia alfa e à detecção de $\mathrm{Hb} \mathrm{H}^{(3,25)}$. Em nosso laboratório também detectamos a talassemia alfa em $5 \%$ dos indivíduos portadores de lúpus eritematoso sistêmico(1).

Já foram descritas algumas drogas capazes de afetar a expressão dos genes das globinas. Fibach et al.(4) (2003) estudaram a indução da produção de $\mathrm{Hb}$ F utilizando mithramycin, droga capaz de se ligar ao DNA, provocando alterações estruturais que resultam em aumento dessa expressão. A cadeia gama foi a que apresentou maior indução com o tratamento; as cadeias alfa e beta também foram induzidas, mas demonstraram menor indução quando comparadas com a cadeia gama.

Viola et al.(21) (2008) apresentaram, utilizando técnica de transcrição reversa quantitativa (Q-RT-PCR), variação em concentração dos mRNA de globinas em células tratadas com psoralenos ou irradiadas com luz ultravioleta (UV). A concentração de mRNA de globina alfa foi maior em células tratadas com 5-metoxipsoraleno (5-MOP) em comparação com células tratadas com 4,5', 4'trimetilangelicina (TMA) ou irradiadas com luz UV. O mRNA de globina gama apresentou mesmo padrão, porém houve menor indução. Portanto, esses dados e os de Fibach et al. ${ }^{(4)}$ (2003) mostram claramente uma indução dos genes globina em resposta ao tratamento com diferentes substâncias.

\section{Conclusão}

Os dados obtidos neste trabalho mostram que a presença de $\mathrm{Hb} \mathrm{H}$ na detecção de talassemia alfa pode não ser confirmada em análise posterior. Entre fatores que podem influenciar o não aparecimento da $\mathrm{Hb} \mathrm{H}$ em pessoa comprovadamente com talassemia alfa está a deficiência de ferro no portador de $\mathrm{Hb} \mathrm{H}$. A talassemia alfa está associada a defeitos genéticos em genes codificadores da cadeia alfa, mas também pode estar relacionada com desbalanciamento temporário na expressão dos genes das globinas (diminuição de alfa ou aumento de beta), o que poderia explicar o aparecimento de tetrâmeros de cadeia beta $(\mathrm{Hb} \mathrm{H})$, sugerindo diagnóstico de talassemia alfa mínima.

É provável que, além de defeitos genéticos associados aos genes alfa, outros fatores possam induzir aparecimento da talassemia alfa. Assim, o diagnóstico dessa doença deve ser tratado com muito cuidado, principalmente em suas formas mais brandas. A presença de $\mathrm{Hb} \mathrm{H}$ deve ser confirmada por diferentes métodos, sendo associada a microcitose e exames realizados em diferentes períodos, devendo, sempre que possível, ser confirmada por métodos de diagnósticos moleculares.

\section{Agradecimentos}

Agradecemos a Fundação de Amparo à Pesquisa do Estado de Goiás (FAPEG) pelo apoio financeiro.

\section{Referências}

1. CASTRO, F. S. et al. Prevalência de talassemias e hemoglobinas variantes em pacientes portadores de lupus eritematoso sistêmico. Rev Bras Hematol Hemoter, v. 30, n. 1, p. 24-8, 2008.

2. CHAN, L. C. et al. Should we screen for globin gene mutations in blood samples with mean corpuscular volume (MCV) greater than $80 \mathrm{fL}$ in areas with a high prevalence of thalassaemia? J Clin Pathol, v. 54, n. 4, p. 317-20, 2001.

3. DOMINGOS, C. R. B. et al. Hemoglobinopatias e hepatoglobinas em portadores de doença de Chagas. Rev Bras Patol Clin, v. 27, n. 3, p. 80-7, 1991.

4. FIBACH, E. et al. Mithramycin induces fetal hemoglobin production in normal and thalassemic human erythroid precursor cells. Blood, v. 102, p. 1276-81, 2003.

5. GOMES, C. T.; NAOUM, P. C.; ÂNGULO, I. L. Talassemia alfa adquirida nas doenças linfo e mieloproliferativas. Rev Bras Patol Clin, v. 24, n. 3, p. 98-101, 1988.

6. JARMAN, A. P. et al. Characterization of the major regulatory element upstream the human alpha-globin gene cluster. Mol Cell Biol, v. 11, n. 9, p. 4679-89, 1991.

7. THOMPSON, C. C. et al. Positional effect of cis/trans alpha globin gene deletions on the formation of " $\mathrm{H}$ " bodies. Am J Hematol, v. 31, n. 4, p. 242-7, 1989.

8. MELO-REIS, P. R. et al. Prevalência de hemoglobinopatias e talassemias em alunos de Biomedicina da UCG. Estudos, Goiânia, v. 31, p. 125-33, 2004. 
9. MELO-REIS, P. R. et al. Prevalência de talassemias e hemoglobinas variantes no estado de Goiás, Brasil. J Bras Patol Med Lab, v. 42, n. 6, p. 425-30, 2006.

10. NAOUM, P. C. Eletroforese, técnicas e diagnósticos. 2. ed. São Paulo: Santos, 1999.

11. SKOGERBOE, K. J. et al. Screening for alpha-thalassemia. Correlation of hemoglobin $\mathrm{H}$ inclusion bodies with DNAdetermined genotype. Arch Pathol Lab Med, v. 116, n. 10, p. 1012-8, 1992.

12. NAOUM, P. C.; BONINI-DOMINGOS, C. R. Dificuldades no diagnóstico laboratorial das hemoglobinopatias. Rev Bras Hematol Hemoter, v. 29, n. 3, p. 226-8, 2007.

13. PUBMED - U. S. National Library of Medicine. Disponível em: <http://www.ncbi.nlm.nih.gov/sites/entrez?db=p ubmed\&cmd=search\&term=thalassemia\%20alpha $>$. Acesso em: 16 set. 2008.

14. PAPAYANNOPOULOS, R.; STAMATONYANNOPOULOS, G. Stains form inclusions bodies. In: Standatization of laboratory reagents and methods for detection of haemoglobinopathies. Atlanta: Hew Publications, 1974.

15. PEREIRA, J. M. et al. Investigação em uma família com alfa talassemia. Rev Brasil Pesqu Med Biol, v. 6, p. 349, 1973.

16. SILVESTRONI, E.; BIANCO, I. Screening for microcytemia in Italy: analysis of collected in the past 30 years. Am J Hum Genet, v. 27, p. 198-212, 1975.
17. STEENSMA, D. P.; GIBBONS, R. J.; HIGGS, D. R. Acquired alpha-thalassemia in association with myelodysplastic syndrome and other hematologic malignancies. Blood, v. 105, p. 443-52, 2005.

18. STEINBERG, M. H. et al. Effects of thalassemia and microcytosis on the hematologic and vasoocclusive severity of sickle cell anemia. Blood, v. 63, p. 1353-60, 1984.

19. TOME-ALVES, R. et al. Hemoglobinas AS/alfa talassemia: importância diagnóstica. Rev Bras Hematol Hemoter, v. 22, n. 3, p. 388-94, 2000.

20. TRENT, R. J. A. Diagnosis of the haemoglobinopathies. Clin Biochem Rev, v. 27, p. 27-38, 2006.

21. VIOLA, G. et al. Induction of $\gamma$-globin mRNA, erythroid differentiation and apoptosis in UVA-irradiated human erythroid cells in the presence of furocumarin derivatives. Bioch Pharmacol, v. 75, p. 810-25, 2008.

22. WEATHERALL, D. J.; CLEGG, J. B. Inherited haemoglobin disorders: an increasing global health problem. WHO, v. 79, n. 8, p. 704-12, 2001.

23. WEATHERALL, D. J.; CLEGG, J. B. The thalassaemia syndromes. 4. ed. Oxford: Blackwell Science Publications, 2001.

24. ZAGO, M. A.; COSTA, F. F. Hereditary hemoglobin disorders in Brazil. Trans Roy Soc Trop Med Hyg, v. 79, n. 3, p. 385-8, 1985.

25. ZAGO, M. A.; FALCÃO, R. P.; PASQUINI, R. Hematologia: fundamentos e prática. São Paulo: Atheneu, 2004.

\begin{tabular}{l|l} 
Endereço para correspondência \\
\hline Karlla Creick Batista Dias-Penna \\
Universidade Católica de Coiás - Departamento \\
de Biomedicina - Área V \\
Avenida Universitária, 1069 - Setor Universitário \\
CEP: 74605-010 - Coiânia-CO \\
Tel.: (62) 3946-1194 \\
e-mail: karllagreick@yahoo.com.br
\end{tabular}

